OPEN ACCESS

Edited by:

Catherine Ayn Brissette, University of North Dakota,

United States

Reviewed by:

Alexandra Rebecca Mey, The University of Texas at Austin,

United States

David Salvador Zamorano Sanchez, Universidad Nacional Autónoma

de México, Mexico

*Correspondence:

Jyl S. Matson

Jyl.Matson@utoledo.edu

Specialty section:

This article was submitted to Microbial Physiology and Metabolism,

a section of the journal

Frontiers in Microbiology

Received: 30 October 2018

Accepted: 22 January 2019

Published: 05 February 2019

Citation:

Saul-McBeth J and Matson JS (2019) A Periplasmic Antimicrobial Peptide-Binding Protein Is Required for Stress Survival in Vibrio cholerae.

Front. Microbiol. 10:161.

doi: 10.3389/fmicb.2019.00161

\section{A Periplasmic Antimicrobial Peptide-Binding Protein Is Required for Stress Survival in Vibrio cholerae}

\author{
Jessica Saul-McBeth and Jyl S. Matson* \\ Department of Medical Microbiology and Immunology, University of Toledo, Toledo, $\mathrm{OH}$, United States
}

Vibrio cholerae must sense and respond appropriately to stresses encountered in the aquatic environment and the human host. One stress encountered in both environments is exposure to antimicrobial peptides (AMPs), produced as a part of the innate immune response by all multicellular organisms. Previous transcriptomic analysis demonstrated

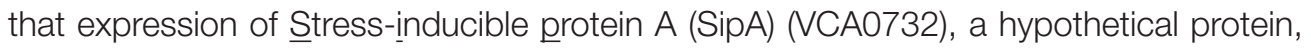
was highly induced by AMP exposure and was dependent on a specific uncharacterized two-component system. In order to better understand role of this protein in stress relief, we examined whether it shared any of the phenotypes reported for its homologs. SipA is required for survival in the presence of two other stressors, cadmium chloride and hydrogen peroxide, and it localizes to the bacterial periplasm, similar to its homologs. We also found that SipA physically interacts with OmpA. Importantly, we found that SipA binds AMPs in the bacterial periplasm. This suggests a model where SipA may act as a molecular chaperone, binding AMPs that enter the periplasm and delivering them to OmpA for removal from the cell. While El Tor $V$. cholerae strains lacking SipA do not show a survival defect in the presence of AMPs, we found that Classical sipA mutants are less able to survive in the presence of AMPs. This phenotype is likely masked in the El Tor background due to a functional lipid A modification system that increases AMP resistance in these strains. In summary, we have identified a protein that contributes to a novel mechanism of stress relief in $V$. cholerae.

Keywords: Vibrio cholerae, cholera, stress response, antimicrobial peptides, outer membrane proteins

\section{INTRODUCTION}

Pathogenic bacteria have evolved to survive in diverse hosts and environments. In order to thrive in these different niches, many bacteria have developed sophisticated mechanisms to alter specific gene expression, resulting in adaptation and survival in the presence of stress. One example of a successful pathogen is Vibrio cholerae, a Gram-negative bacterium that causes the diarrheal disease cholera. Not only is $V$. cholerae able to alter its gene expression quickly in response to the aquatic environment, with fluctuating salinity, $\mathrm{pH}$, and nutrient availability, but it can also survive stresses in the human gut, where it may encounter reactive oxidative species, competing commensal bacteria, and other stressors (Joelsson et al., 2007). One of the stresses $V$. cholerae must overcome to successfully colonize the human intestine is antimicrobial peptides (AMPs) produced by intestinal epithelial cells as a part of innate immunity. 
Antimicrobial peptides are small, positively charged peptides that have both hydrophobic and hydrophilic residues near the N-termini, which enable them to interact with both the aqueous environment and lipid-rich membranes (Izadpanah and Gallo, 2005). AMPs are produced by bacteria, archeaea, and eukarya (including mammals), and are able to either lyse or prevent replication of a broad spectrum of organisms including bacteria, viruses, and parasites (Bahar and Ren, 2013). There are two main groups of AMPs produced by the human host: cathelicidins and defensins. LL-37, the only human member of the cathelicidin family, contains an $\mathrm{N}$-terminal signal peptide and can function both as an antimicrobial and as a chemoattractant (Durr et al., 2006). Polymyxin B, another example of a cationic AMP, is derived from the soil bacterium Bacillus polymyxa (Brownlee and Jones, 1948). Historically, polymyxin B has been used to clinically differentiate the Classical and El Tor biotypes of $V$. cholerae, and has also been widely used in studies on bacterial stress. Many AMPs, including LL-37 and polymyxin $\mathrm{B}$, are thought to act on Gram-negative bacteria by making initial non-specific interactions with the membrane through electrostatic interactions with the negatively charged lipopolysaccharide (LPS) layer (Izadpanah and Gallo, 2005; Sorensen et al., 2008). Due to their amphipathic structure, cationic AMPs can enter the membrane by forming pores or channels (Izadpanah and Gallo, 2005). Once inside the cell, through not clearly defined mechanisms, both polymyxin B and LL-37 can enter the periplasm and cytoplasm where they may interfere with DNA and protein synthesis (Choi et al., 2017).

Due to the potential lethality of AMPs, $V$. cholerae has developed a range of strategies to overcome AMP damage including: the production of outer membrane vesicles (OMVs) to titrate the AMPs, changes in porin composition of the outer membrane to modify permeability, the use of efflux pumps to export AMPs, LPS modifications to prevent AMP binding, and two-component systems (TCS) that transcriptionally regulate all of these mechanisms (Nizet, 2006; McBroom and Kuehn, 2007; Band and Weiss, 2015). In V. cholerae and other Gram-negative bacteria, the outer surface is composed of LPS, which acts as the first line of defense against extracellular stresses, including AMPs. Components of the LPS, particularly the lipid A and core oligosaccharide, are negatively charged, allowing AMPs to bind easily. However, V. cholerae encodes a lipid A modification system that alters the charge of the LPS, reducing AMP binding and subsequent entry into the bacterial cell. This modification system is encoded by the almEFG gene cluster, and alters the LPS through addition of a glycine or diglycine to lipid A (Henderson et al., 2014; Herrera et al., 2014). Interestingly, Classical strains of $V$. cholerae contain a frameshift mutation in $\operatorname{almF}$, resulting in a non-functional modification system (Henderson et al., 2014). This explains why classical $V$. cholerae strains are far more sensitive to AMPs than El Tor strains (Matson et al., 2010; Henderson et al., 2014, 2017).

Two-component systems are important regulators of stress response mechanisms in bacteria. They are composed of a sensor kinase, located in the inner membrane, that senses a change in the external environment, and the response regulator, located in the cytoplasm, that responds accordingly by activating a specific set of genes. $V$. cholerae encodes TCSs that respond to AMP stress, including CarRS (VprAB) (Herrera et al., 2010, 2014; Bilecen et al., 2015; Henderson et al., 2017). In a previous study, we aimed to determine the function of a putative TCS (VC1638/39) that resembles PhoPQ in Salmonella enterica sv. Typhimurium in domain structure and conserved residues (Matson et al., 2017). In that study, RNA-Seq was used to identify genes differentially regulated by this TCS in the presence and absence of sublethal concentrations of polymyxin B (Matson et al., 2017). One of the interesting results concerned VCA0732, a $15 \mathrm{kDa}$ hypothetical protein that belongs to a subfamily of the bacterial OB-fold family of proteins (Ginalski et al., 2004). Bacterial OB-fold proteins are characterized by a conserved oligonucleotide/oligosaccharidebinding (OB) fold domain which forms a binding pocket and is predicted to bind positively charged ligands and small molecules (Ginalski et al., 2004). We found that polymyxin B exposure highly induces vca0732 expression, and that VC1638 and VC1639 are required for its expression (Matson et al., 2017). We hypothesized that VCA0732 must play a role in responding to the stress of AMP exposure and that the TCS was required to regulate this response. Puzzlingly, a vca0732 mutant strain showed no survival defect in the presence of polymyxin B, making the role of VCA0732 in the AMP stress response unclear.

In the present study, we aimed to better understand the function of VCA0732 and to characterize its role in responding to stress in V. cholerae. We first examined whether or not VCA0732 functioned similarly to its known homologs in other bacteria. We found that VCA0732 has several similarities to its homologs, including periplasmic localization and its role in relieving stress caused by other molecules. VCA0732 also interacts with an outer membrane protein, OmpA, similar to its homologs. In addition, we found that VCA0732 directly binds AMPs both in vivo and in vitro. This suggests a model where VCA0732 (and its homologs) may function to chaperone AMPs to porins, positioning them for efflux out of the cell. While a vca0732 mutant is insensitive to AMP killing in El Tor V. cholerae, we found that Classical strains require VCA0732 for survival. This suggests that the presence of the lipid A modification system in El Tor strains masks the survival phenotype observed in the Classical background. Taken together, these findings demonstrate that VCA0732 is a part of a previously unappreciated stress response mechanism and that it plays an important role in protecting $V$. cholerae from AMP killing. Due to the observed roles of VCA0732 in responding to AMPs and other stressors, we

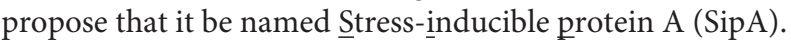

\section{MATERIALS AND METHODS}

\section{Bacterial Strains and Growth Conditions}

All bacterial strains were grown at $37^{\circ} \mathrm{C}$ with agitation in Lysogeny Broth (LB) or on LB agar plates. Strains and plasmids used in this study are listed in Supplementary Table S1. Strains were derived from V. cholerae C6706 and O395 or E. coli K-12 strain BW25113. Plasmids used in this study were pKAS32, pTL61T, pBAD18-Kan and pBAD18-Cam (Linn and St Pierre, 1990; Guzman et al., 1995; Skorupski and Taylor, 1996). 
Antibiotics were used at the following concentrations: polymyxin B, $40 \mu \mathrm{g} / \mathrm{mL}$ for El Tor and $10 \mu \mathrm{g} / \mathrm{mL}$ for Classical; LL-37, $10 \mu \mathrm{g} / \mathrm{mL}$ for El Tor and $5 \mu \mathrm{g} / \mathrm{mL}$ for Classical; streptomycin, $100 \mu \mathrm{g} / \mathrm{mL}$; kanamycin, $50 \mu \mathrm{g} / \mathrm{mL}$; ampicillin, $100 \mu \mathrm{g} / \mathrm{mL}$; chloramphenicol, $5 \mu \mathrm{g} / \mathrm{mL}$. Arabinose was used at $0.2 \%$ to induce expression from the $\mathrm{pBAD}$ plasmids.

\section{Strain Construction}

The C6706 $\Delta$ sipA ( $\Delta v c a 0732)$ strain used in this study has been described previously (Matson et al., 2017). The chromosomally His-tagged SipA strain was constructed using SOE-ing PCR (Innis, 1990). Briefly, 500 bp upstream and downstream of the sipA coding region were amplified separately using primers containing a $6 \times$ His sequence prior to the stop codon. After SOE-ing, the resulting PCR product was digested with XbaI and SacI and ligated into the suicide plasmid pKAS32 (Skorupski and Taylor, 1996). The resulting recombinant plasmid was

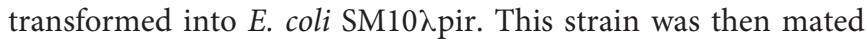
with $V$. cholerae strain C6706. Integration of the plasmid into the $V$. cholerae chromosome was selected for by plating on TCBS (thiosulfate-citrate-bile-sucrose, Difco) containing $50 \mu \mathrm{g} / \mathrm{mL}$ ampicillin (Matson and DiRita, 2005) and conjugation was performed as previously described (Beck et al., 2004). O395 $\Delta$ sipA and $\triangle o m p A$ were constructed by amplifying 500 bp segments upstream and downstream of the coding regions containing a internal deletion of the sipA or ompA gene. The products were joined together via SOE-ing PCR, cloned into suicide vector pKAS32, and transformed into E. coli as above (Skorupski and Taylor, 1996). The constructs were then conjugated into O395 $V$. cholerae as above and isolates containing the gene deletion were identified using PCR.

\section{Plasmid Construction}

FLAG-tagged Crp, OmpA, and Tsp constructs were generated by amplification of the coding region of each gene from $V$. cholerae C6706 (Crp, OmpA) or O395 (Tsp) chromosomal DNA, using primers that added the C-terminal FLAG sequence with Radiant HiFi Ultra polymerase (Alkali Scientific Inc.). The coding sequence of $y g i W$ was amplified from E. coli K-12. The sipA coding sequence was amplified from C6706. After amplification, the $y g i W$, ompA, and sipA PCR products were digested with SacI and $X b a I$ and ligated into the arabinose-inducible expression vector pBAD18-Kan (Guzman et al., 1995). The crp construct was digested with SacI and XbaI and ligated into pBAD18-Cam. All constructs were confirmed by sequencing.

\section{Protein Purification}

Proteins were purified according to Huntley et al. (2007) with a few modifications. E. coli strain JM101 containing plasmid constructs encoding SipA- $6 \times$ his or VC1638- $6 \times$ his was grown in LB to an optical density (OD) of $\sim 0.4$, after which protein expression was induced for $4 \mathrm{~h}$ by the addition of $0.2 \%$ arabinose. Following induction, cells were collected by centrifugation and frozen overnight at $-20^{\circ} \mathrm{C}$. The following day, pellets were thawed and resuspended in $30 \mathrm{~mL}$ of $10 \mathrm{mM}$ Tris, $500 \mathrm{mM}$ $\mathrm{NaCl}, 10 \mathrm{mM}$ imidazole, and complete mini EDTA free protease inhibitors (Qiagen). The cell suspension was freeze-thawed $3 \times$, followed by removal of cellular debris by centrifugation. The resulting supernatant was applied to a column of pre-equilibrated HisPur Ni-NTA Resin (Thermo Fisher Scientific) followed by washing. Purified recombinant proteins were then eluted using lysis buffer containing $200 \mathrm{mM}$ imidazole and dialyzed into $10 \mathrm{mM}$ Tris, $100 \mathrm{mM} \mathrm{NaCl}$, and $20 \%$ glycerol. Protein quantification was performed using a Qubit 2.0 fluorometer (Invitrogen).

\section{Enzyme-Linked Immunosorbent Assay (ELISA)}

Ninety-six-well plates were coated with purified SipA or VC1638, at a concentration of $10 \mu \mathrm{g} / \mathrm{mL}$ overnight at $4^{\circ} \mathrm{C}$ in binding buffer (30 mM Na $2 \mathrm{CO}_{3}, 69 \mathrm{mM} \mathrm{NAHCO}$ ) (Lin et al., 2015). Plates were washed with PBS-T $3 \times$ and blocked for $1 \mathrm{~h}$ in $5 \%$ bovine serum albumin (BSA) + PBS-T at RT. Plates were washed and either LL-37 or biotinylated polymyxin B were added at $10 \mu \mathrm{g} / \mathrm{mL}$ and incubated for $1 \mathrm{~h}$. Plates were washed again and anti-LL-37 (mouse; Santa-Cruz Biotechnology), was added for $1 \mathrm{~h}$. After washing, either Goat pAb to Mouse IgG + IgM HRP (Abcam), or Strepavidin-HRP (Abcam) was added. TMB (Tetramethylbenzidine; Fisher Scientific) was used for detection and the reaction was stopped using $2 \mathrm{~N} \mathrm{HCl}$. The colorimetric change was measured at $\mathrm{OD}_{450}$. Experiments were performed in triplicate and $P$ values represent one experiment containing technical triplicates. Significance was determined using ANOVA with Tukey's post hoc test.

\section{Survival Assays}

Bacterial cultures were grown to an $\mathrm{OD}_{600}$ of $\sim 0.5$ prior to adding cadmium chloride, hydrogen peroxide, polymyxin B, or LL-37. Aliquots of $900 \mu \mathrm{L}$ of cells were incubated with $100 \mu \mathrm{L}$ freshly prepared hydrogen peroxide at $2 \mu \mathrm{M}$ for $V$. cholerae and $34 \mathrm{mM}$ for $E$. coli for $15 \mathrm{~min}$ (Lee et al., 2010). Cadmium chloride was used at $200 \mu \mathrm{M}$ for $E$. coli and $150 \mu \mathrm{M}$ for $V$. cholerae for $40 \mathrm{~min}$. Classical $V$. cholerae was treated with $10 \mu \mathrm{g} / \mathrm{mL}$ polymyxin B or $5 \mu \mathrm{g} / \mathrm{mL} \mathrm{LL}-37$ for $1 \mathrm{~h}$. After treatment, samples were serially diluted and plated for enumeration. The percent survival was determined by dividing the CFU of treated samples by those of untreated samples $\times 100$. Assays were performed in triplicate and $P$ values were calculated from all three experiments using ANOVA with Tukey's post hoc test.

\section{$\beta$-Galactosidase Assays}

Vibrio cholerae strains were grown overnight at $37^{\circ} \mathrm{C}$, then subcultured 1:50 into LB and grown for $4 \mathrm{~h}$ with aeration. Samples were placed on ice and chloramphenicol was added at $0.5 \mathrm{mg} / \mathrm{mL}$. $\beta$-galactosidase assays were performed according to Miller (1972).

\section{Cellular Fractionation and Immunodetection}

Bacterial fractionation was performed according to Petiti et al. (2017) with a few modifications. C6706, C6706 containing TspFLAG or CRP-FLAG, or C6706 containing chromosomally Histagged SipA was grown for $3 \mathrm{~h}$ with aeration. Polymyxin B was 
added at $40 \mu \mathrm{g} / \mathrm{mL}$ to induce SipA expression for $1 \mathrm{~h}$. $0.2 \%$ arabinose was added to induce expression of Tsp-FLAG and CRPFLAG. Whole-cell lysates were prepared by centrifugation and suspension of the pellet in $500 \mu \mathrm{L}$ of Radioimmunoprecipitation assay buffer (RIPA) (25 mM Tris pH 8.0, $150 \mathrm{mM} \mathrm{NaCl}, 0.1 \%$ SDS, $0.5 \%$ sodium deoxycholate, $1 \%$ NP-40, and $10 \mathrm{mM} \mathrm{PMSF}$ ). The lysate was then subjected to 3 freeze-thaw cycles, followed by centrifugation and collection of $200 \mu \mathrm{L}$ of supernatant. The periplasmic fraction was isolated by centrifuging bacterial cells, washing $3 \times$ with $1 \times$ PBS, and suspension of the pellet by inversion in $200 \mu \mathrm{L}$ TES buffer $(0.5 \mathrm{M}$ sucrose, $0.5 \mathrm{mM}$ EDTA, $200 \mathrm{mM}$ Tris-HCl, pH 8.0). After careful mixing, $720 \mu \mathrm{L} 2 \times$ diluted TES buffer containing $10 \mathrm{mg} / \mathrm{mL}$ lysozyme was added and incubated on ice for $30 \mathrm{~min}$. Following centrifugation for $30 \mathrm{~min}$ at max speed, $200 \mu \mathrm{L}$ of the supernatant was collected, representing the periplasmic fraction. The pellet was resuspended in $720 \mu \mathrm{L}$ of $2 \times$ diluted TES buffer containing $2 \mathrm{mM} \mathrm{MgCl}_{2}$ and lysed by $3 \times$ freeze-thaw cycles, followed by centrifugation and collection of the supernatant. The supernatant was then centrifuged at $100,000 \times g$ for $45 \mathrm{~min}$ and $200 \mu \mathrm{L}$ of supernatant was collected, representing the cytoplasmic fraction. All fractions were precipitated with $10 \%$ TCA for $30 \mathrm{~min}$ on ice. Samples were washed with $1 \mathrm{~mL}$ acetone, centrifuged, resuspended in SDS-loading buffer, and boiled for $5 \mathrm{~min}$. Proteins were separated using 15\% (wt/vol) polyacrylamide gels, transferred to nitrocellulose membranes and probed with either His HRP-labeled Mouse Monoclonal IgG (R\&D Systems) or OctA-Probe (H-5) HRP (Santa-Cruz Biotechnology). The protein fractionation controls used were: Tsp-FLAG (periplasm) and CRP-FLAG (cytoplasm).

\section{Outer Membrane Protein (OMP) Isolation (ROMP)}

Outer membrane proteins were isolated via rapid outer membrane protein procedure (ROMP) (Carlone et al., 1986). Strains containing recombinant protein were grown and induced for $4 \mathrm{~h}$. After growth, samples were centrifuged and pellets were resuspended in $1 \mathrm{~mL} 10 \mathrm{mM}$ HEPES, $\mathrm{pH}$ 7.4. Lysis was performed by repeated freeze-thaw cycles. Cellular debris was removed by centrifugation at $15,600 \times g$ for $5 \mathrm{~min}$ at $4^{\circ} \mathrm{C}$. The supernatant was then centrifuged at $15,600 \times g$ at $4^{\circ} \mathrm{C}$ for $30 \mathrm{~min}$ to pellet the cell membrane fraction. The supernatant was removed and the membrane-containing pellet was resuspended in $0.2 \mathrm{~mL} 10 \mathrm{mM}$ HEPES, $\mathrm{pH}$ 7.4. The membranes were solubilized by the addition of $0.2 \mathrm{~mL} 2 \%$ sarkosyl in $10 \mathrm{mM}$ HEPES, $\mathrm{pH}$ 7.4, and incubated on ice for 30 min with intermittent pipetting. The outer membrane was then pelleted by centrifugation as described above and washed with $10 \mathrm{mM}$ HEPES. After washing, the pellet was resuspended in SDS-PAGE loading buffer.

\section{Far-Western Blotting}

Outer membrane protein fractions from C6706 expressing OmpA-FLAG were serially diluted and separated by SDSPAGE. The far western protocol is based on Chen, et al. with modifications ( $\mathrm{Wu}$ et al., 2007). After separation by electrophoresis, the proteins were transferred to a nitrocellulose membrane (GE Healthcare) and the membrane was blocked in $5 \%$ milk in PBST for $1 \mathrm{~h}$. The membrane was then incubated with purified His-tagged SipA at $10 \mu \mathrm{g} / \mathrm{mL}$ overnight at $4^{\circ} \mathrm{C}$. After incubation, SipA was detected via His HRP-labeled Mouse Monoclonal $\operatorname{IgG}_{1}$ (R\&D Systems).

\section{Immunoprecipitation}

C6706 or C6706 4 sipA containing a SipA plasmid was grown for $3 \mathrm{~h}$ in LB with shaking, and $10 \mu \mathrm{g} / \mathrm{mL}$ LL-37 was added for $1 \mathrm{~h}$ before pelleting by centrifugation. The pellet was then resuspended in $500 \mu \mathrm{L} 1 \times$ PBS containing $1 \mathrm{mM}$ DSP (Dithiobis Succinimidyl Propionate; Pierce) to crosslink proteins, and incubated on ice for $30 \mathrm{~min}$. The reactions were quenched by adding $50 \mathrm{mM}$ Tris- $\mathrm{HCl}, \mathrm{pH} 8.0$ for $15 \mathrm{~min}$ on ice, and the samples were centrifuged at $4^{\circ} \mathrm{C}$. The resulting pellet was resuspended in $500 \mu \mathrm{L}$ RIPA buffer and subjected to $3 \times$ freezethaw lysis. After centrifugation at $4^{\circ} \mathrm{C}, 50 \mu \mathrm{L}$ of supernatant was collected and used as the input control. The remaining $450 \mu \mathrm{L}$ of supernatant was added to $40 \mu \mathrm{L}$ HisPur Ni-NTA equilibrated in RIPA buffer and rotated at $4^{\circ} \mathrm{C}$ for $1 \mathrm{~h}$. Samples were centrifuged at $2000 \times g$ for $2 \mathrm{~min}$ and washed in RIPA buffer $3 \times$ before resuspending in SDS-loading buffer and boiling for 15 min. SDS-PAGE and western blotting for LL-37 and SipAHis were performed as above. For identification of potential SipA interaction partners, C6706 containing a SipA plasmid was grown, crosslinked, and immunoprecipitated in the same manner (in the absence of LL-37). Proteins in the elution were visualized by Coomassie staining, excised from the gel, and identified by mass spectrometry (MS Bioworks, Ann Arbor, MI, United States).

Strains containing both the OmpA-FLAG and SipA- $6 \times$ his constructs, or OmpA-FLAG construct and empty vector, were grown and protein expression was induced for $1 \mathrm{~h}$ at $37^{\circ} \mathrm{C}$. The cells were centrifuged and crosslinked with DSP as above. Cells were lysed in buffer containing 0.5M HEPES, $2 \mathrm{M} \mathrm{MgCl}_{2}, 10 \mathrm{~mL}$ $1 \mathrm{M} \mathrm{KCl}, 10 \%$ glycerol, $1 \%$ Triton-X, and $20 \mathrm{mM}$ imidazole (Wu et al., 2007) by $3 \times$ freeze-thaw. Cellular debris was removed by centrifugation and the supernatant was added to HisPur Ni-NTA beads and rotated overnight at $4^{\circ} \mathrm{C}$. Immunoprecipitation and western blotting were performed as above.

\section{RESULTS}

\section{A V. cholerae sipA Mutant Is Sensitive to Cadmium Chloride and Hydrogen Peroxide Stress}

To understand the role of SipA in stress resistance, we wanted to determine if it shared any of the known phenotypes or functions with its homolog from Escherichia coli, YgiW (Lee et al., 2010). Since YgiW is required for survival in the presence of cadmium chloride and hydrogen peroxide stress (Lee et al., 2010), we examined the survival of an El Tor $V$. cholerae sipA deletion strain in the presence of these compounds. We found that when exposed to hydrogen peroxide, a sipA mutant strain showed a significant defect in survival (Figure 1A). We saw a similar survival defect in the presence of cadmium chloride (Figure 1B). 
A

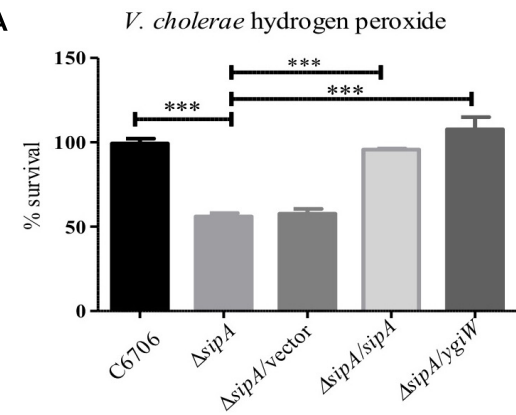

C

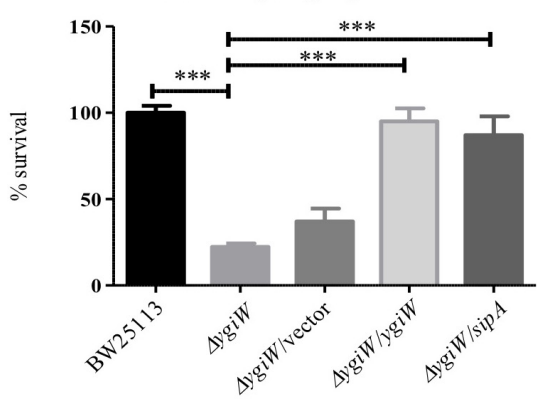

B V. cholerae cadmium chloride

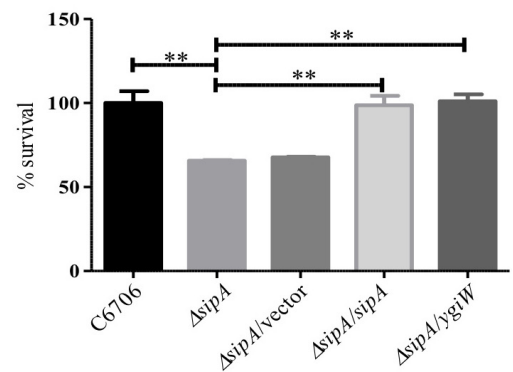

D

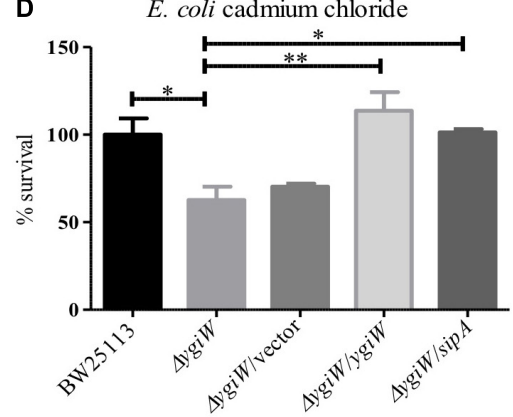

FIGURE 1 | SipA is required for survival in the presence of cadmium chloride and hydrogen peroxide. V. cholerae (A,B) and E. coli (C,D) were grown to mid-logarithmic phase and then treated with: (A) $2 \mu \mathrm{M}$ hydrogen peroxide for $15 \mathrm{~min}$, (B) $150 \mu \mathrm{M}$ cadmium chloride for $45 \mathrm{~min}$, (C) $34 \mathrm{mM}$ hydrogen peroxide for $15 \mathrm{~min}$, and (D) $200 \mu \mathrm{M}$ cadmium chloride for $45 \mathrm{~min}$. After treatment, serial dilutions of each culture were plated for enumeration. Data represents the means and standard deviations of at least three samples. ${ }^{*} p=<0.05,{ }^{* *} p=<0.01,{ }^{* * *} p<0.001$.

Expression of SipA from a plasmid was able to restore wild type resistance to both compounds. In addition, we also tested whether the $y g i W$ gene from E. coli could complement these phenotypes in the $V$. cholerae background, and found that it also restored survival to wild type levels (Figures 1A,B). Finally, we obtained a $y g i W$ mutant $E$. coli strain [Keio collection (Baba et al., 2006)] and tested both ygiW and sipA for complementation in the E. coli background. Again, both homologs were able to restore resistance to hydrogen peroxide and cadmium chloride to wild type levels (Figures 1C,D). Overall, these results show that both $y g i W$ and sipA complement and cross-complement the resistance phenotypes in both bacterial species (Figures 1A-D). This suggests that SipA in V. cholerae plays a role in stress relief and has a similar cellular function as YgiW in E. coli under the conditions tested.

Our previous work showed that AMP stress induces expression of sipA (Matson et al., 2017). We therefore wanted to determine if these two other stressors also induce its expression. A sipA-lacZ reporter fusion was used to assess sipA promoter activity in the presence of cadmium chloride and hydrogen peroxide. While both compounds clearly induced sipA expression in $V$. cholerae, it was to a lower level than the induction by polymyxin B (Supplementary Figure S1).

\section{SipA Localizes to the Periplasm}

The known homologs of SipA, YgiW (E. coli) and YdeI (Salmonella enterica), localize to the periplasmic space (Ginalski et al., 2004; Pilonieta et al., 2009). In addition, subcellular localization prediction tools suggest that SipA is a periplasmic protein (data not shown). In order to determine whether SipA localizes to the periplasm of $V$. cholerae we performed cellular fractionation studies. These studies were performed in the presence and absence of polymyxin B in order to determine whether or not the antimicrobial peptide influences localization of the protein, in addition to its established role in sipA expression (Matson et al., 2017). We first generated a strain of $V$. cholerae where sipA was chromosomally His-tagged. This allowed us to detect SipA using anti-His antiserum, and avoid any localization artifacts that could be introduced by overexpression of the protein. We co-expressed FLAG-tagged Tsp and FLAG-tagged CRP in this background to use as periplasmic and cytoplasmic control proteins, respectively. Tailspecific protease (Tsp) is a periplasmic protease that degrades the virulence regulator TcpP when conditions do not favor virulence gene expression (Teoh et al., 2015). CRP (cAMP receptor protein) is a cytoplasmic protein that functions as a global transcriptional regulator. Upon separation into the cytoplasmic and periplasmic fractions, the majority of SipA localized to the periplasmic compartment, similar to the known periplasmic protein, Tsp (Figure 2). In the absence of polymyxin B, too little SipA was expressed for detection of the protein, consistent with the previous observation that its expression is highly induced by polymyxin B exposure (Matson et al., 2017). This demonstration that SipA localizes to the periplasmic space, similar to its homologs, suggests that its functional role occurs in that cellular compartment. 


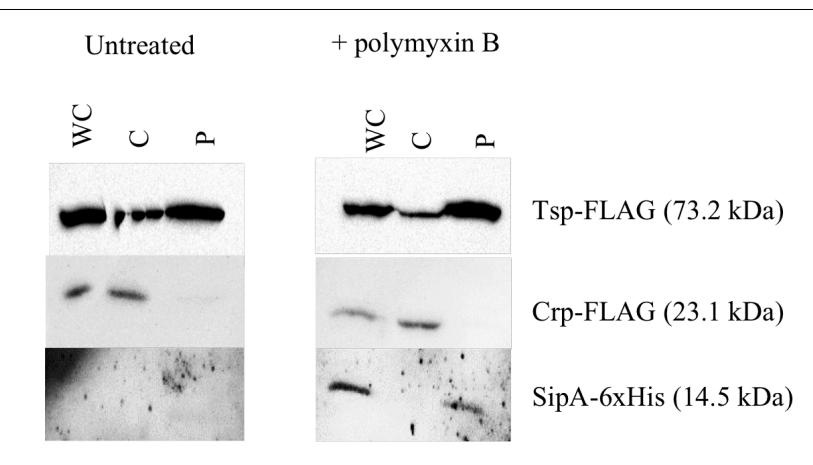

FIGURE 2 | SipA localizes to the periplasmic space. Immunoblotting of whole-cell lysates (WC) and soluble fractions from a $V$. cholerae strain containing a chromosomal $6 \times$ his-tagged $\operatorname{sip} A$. The membrane was probed with anti-FLAG and anti-His antibodies. Tsp-FLAG and Crp-FLAG were used as markers for the periplasmic $(P)$ and cytoplasmic $(C)$ fractions, respectively.

\section{SipA Physically Interacts With OmpA}

Previous studies on the SipA homologs showed that they interact with outer membrane proteins (OMPs). YgiW interacts with OmpF in E. coli, while YdeI interacts with OmpD in S. enterica (Pilonieta et al., 2009). Therefore, we were interested in determining if SipA also interacted with an OMP in $V$. cholerae. Initially, to identify potential interaction partners, SipA was overexpressed from a plasmid and used to coimmunoprecipitate proteins after crosslinking. Bound proteins were identified using mass spectrometry. Although other studies hypothesized that SipA may interact with OmpU (Pilonieta et al., 2009), no $\mathrm{OmpU}$ fragments were identified in this pilot study. However, OmpA fragments were found (data not shown). To determine if SipA physically interacts with OmpA, we first performed farwestern analysis. $V$. cholerae overexpressing FLAG-tagged OmpA was fractionated to isolate the outer membrane (OM) fraction. Increasing concentrations of OM fraction were separated by SDSPAGE, transferred to a nitrocellulose membrane, and probed with purified His-tagged SipA. After incubation, bound SipA was detected using anti-His antibodies. We also performed an identical experiment in wild type cells expressing native levels of OmpA. We observed that, with increasing amounts of loaded protein, increased SipA was detected binding to the blot (Figure 3A). Importantly, the observed bands corresponded to the molecular weight of OmpA $(35.5 \mathrm{kDa})$, which we could detect using an anti-FLAG antibody in the samples that were also subjected to far-western blotting (Supplementary Figure S2). We also observed faint bands corresponding to the size of OmpA in the wild type background. In addition, we detected bands of a lower molecular weight in both backgrounds. This could either represent a degradation product of OmpA that cannot be detected using the FLAG antibody or another potential interaction partner that remains to be identified.

To determine if OmpA interacts with SipA in vivo, we performed coimmunoprecipitation studies. His-tagged SipA was co-expressed in $V$. cholerae also containing a plasmid expressing FLAG-tagged OmpA. As a control, we also performed the coimmunoprecipitation in a $\operatorname{sipA}$ deletion strain. Cellular proteins were crosslinked using DSP, and the cells were lysed and applied to a nickel-NTA column. Prior to loading onto the column, both strains produced detectable OmpA and only the SipA-expressing strain produced SipA (input; Figure 3B). In the eluate from the column (output), OmpA was only detected in the strain expressing SipA, suggesting that the two proteins interact in the bacterial cell. OmpA was not detected in the eluate from the control, indicating that OmpA was binding to SipA and was not binding non-specifically to the Ni-NTA resin (Figure 3B). These data suggest that OmpA is an interaction partner of SipA and provides additional evidence that there may be a functional connection between this subfamily of proteins and OMPs.

\section{SipA and OmpA Likely Function in the Same Biological Pathway}

Based on our observation that SipA and OmpA interact in $V$. cholerae, we wanted to determine if OmpA contributes to the stress resistance phenotypes we had observed for SipA. We have already shown that a strain lacking $\operatorname{sip} A$ is sensitive to hydrogen peroxide and cadmium chloride (Figure 1). We predicted that, if OmpA contributes to hydrogen peroxide and cadmium chloride stress relief in conjunction with SipA as part of a shared mechanism, an ompA mutant would also be similarly sensitive to both compounds. To that end, we generated an ompA deletion strain and assayed its survival in the presence of hydrogen peroxide and cadmium chloride (Figure 4). We found that the ompA deletion showed similar sensitivity as a sipA deletion. Additionally, one would expect that, if the two genes function in the same pathway, a double deletion would neither show an additive phenotype, nor significantly reduced survival. When we performed survival assays in a $V$. cholerae strain lacking both sipA and ompA we did not observe any further sensitivity to either compound (Figure 4). These results suggest that SipA and OmpA likely function in the same stress resistance pathway.

\section{SipA Interacts With Antimicrobial Peptides}

The function of the SipA homologs YdeI and YgiW is not fully understood, however, it has been hypothesized that YdeI could act as a chaperone that shuttles AMPs to the OMP OmpD for removal from the cell in S. Typhimurium (Pilonieta et al., 2009). However, interaction between AMPs and the SipA homologs has not been demonstrated previously. To determine if SipA could perform such a function, we tested whether or not SipA directly interacts with AMPs. We first tested this possibility in vitro using an enzyme-linked immunosorbent assay (ELISA) (Lin et al., 2015). To that end, His-tagged SipA was purified and used to coat wells of a 96-well plate. Then, either LL-37 or biotinylated polymyxin B was added. Antibodies were used to detect LL-37 (HRP conjugated) or biotin-labeled polymyxin B (Streptavidin-HRP). As a negative control, we also assessed AMP binding to VC1638, a protein that is not predicted to interact with AMPs. Significantly higher binding of both LL37 and polymyxin B to SipA was observed compared to the control protein (Figures 5A,B). In addition, due to the fact that the polymyxin B was biotin-labeled for detection purposes, 
A

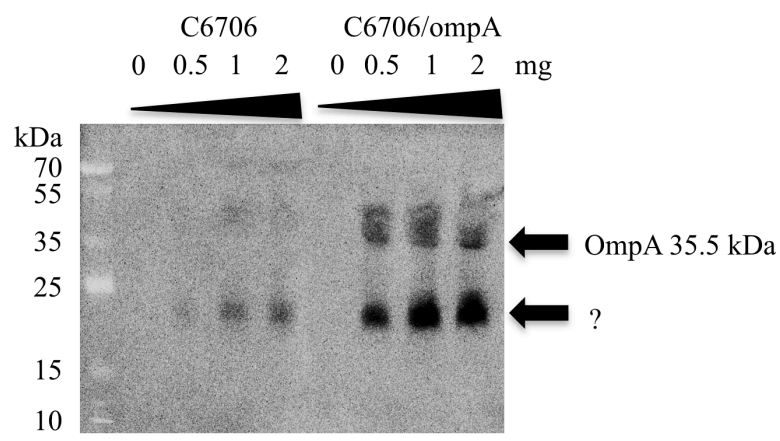

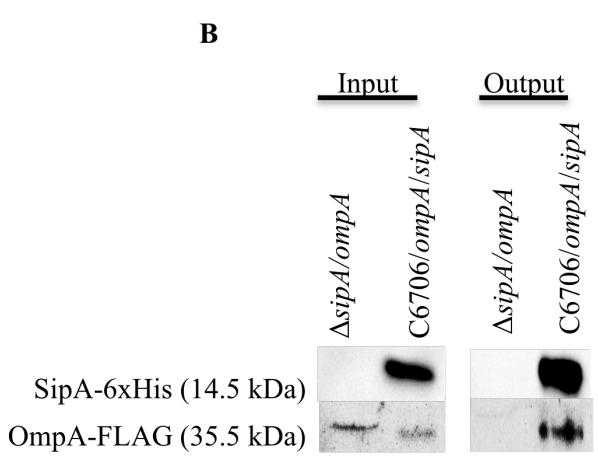

FIGURE 3 | SipA interacts with OmpA. (A) C6706 with and without overexpressed OmpA was grown to an $\mathrm{OD}_{600}$ of 0.5. Outer membranes were isolated, and a range of isolated total outer membrane proteins $(0,0.5,1$, and $2 \mathrm{mg}$ ) was separated by SDS-PAGE and transferred to a membrane. Purified $6 \times$ his-SipA was incubated with the membrane overnight and detected using anti-6xHis. Results are representative of at least three independent experiments. (B) sipA mutant $V$. cholerae overexpressing OmpA-FLAG and C6706 overexpressing OmpA-FLAG and SipA-6xHis were grown to an OD600 of 0.5. Samples were crosslinked with $1 \mathrm{mM}$ DSP and coimmunoprecipitation was performed via Ni-NTA beads. Samples were analyzed by SDS-PAGE and western blotting and detected using anti-FLAG and anti-His antibodies.

\section{A}

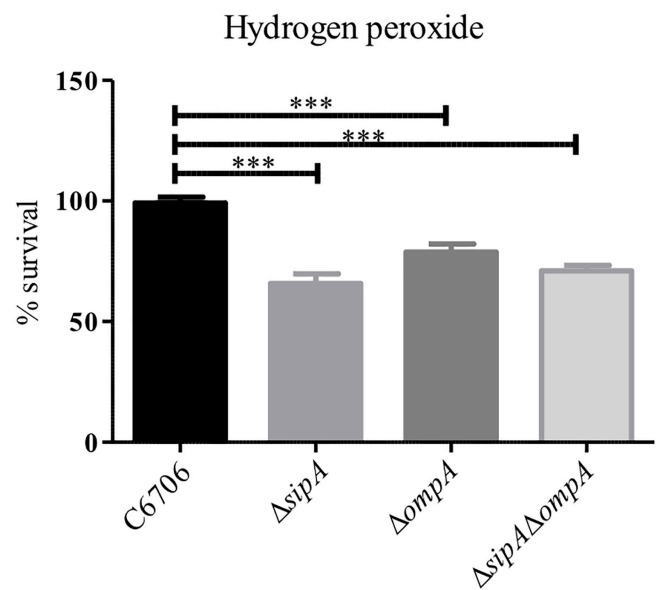

B

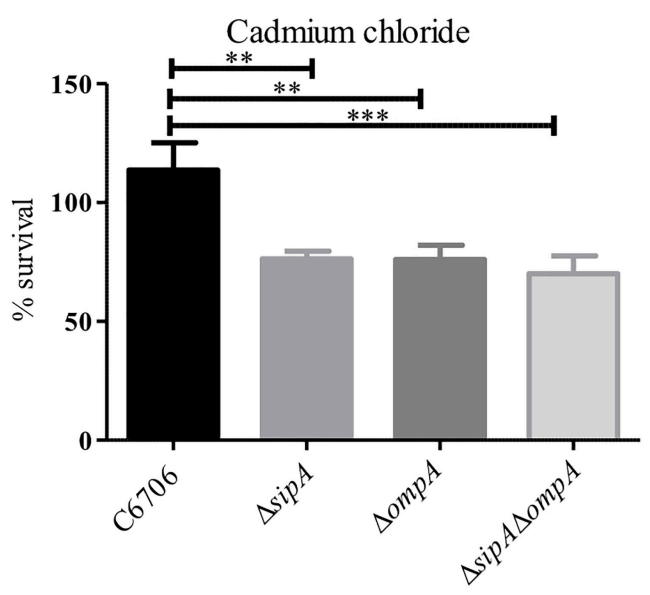

FIGURE 4 | SipA and OmpA likely function in the same pathway. C6706, sipA mutant, ompA mutant, and a sipA ompA double mutant were grown to mid-logarithmic phase and treated with either (A) $2 \mu \mathrm{M}$ hydrogen peroxide for 15 min or (B) $150 \mu \mathrm{M}$ cadmium chloride for 45 min. After treatment, serial dilutions of each culture were plated for enumeration. Data represents the means and standard deviations of at least three samples. ${ }^{* *} p=<0.01,{ }^{* * *} p<0.001$.

we were also able to test whether the addition of excess unlabeled polymyxin specifically competed with the labeled polymyxin B for SipA binding (Figure 5B). The addition of unlabeled polymyxin $\mathrm{B}$ eliminated the binding of the biotinlabeled polymyxin $\mathrm{B}$, indicating that the interaction observed between polymyxin B and SipA was direct and not due to the biotin label.

To further examine the interaction between SipA and AMPs we performed coimmunoprecipitation studies in bacterial cells. sipA mutant $V$. cholerae either containing an empty vector or a sipA overexpression plasmid was grown in the presence of LL37. Cellular proteins were crosslinked, and the amount of LL-37 associated with the bacteria was assessed by immunoblotting. We observed an equivalent amount of LL-37 in the whole cell lysates prior to loading onto the nickel-NTA resin (input, Figure 5C). After bound proteins were eluted from the nickel column, we detected a much larger amount of LL-37 in the eluate from the SipA-expressing sample compared to $\Delta$ sipA strain (Figure $5 \mathrm{C}$ ). This suggests that LL-37 can also interact with SipA in vivo. This is the first evidence that members of this family of proteins bind to AMPs.

For the observed interaction between LL-37 and SipA to be biologically relevant, it would require that AMPs gain access to the periplasmic space of $V$. cholerae, where SipA is likely to perform its cellular function. Previous studies showed that LL37 can enter the periplasm of E. coli (Sochacki et al., 2011), but this had not been tested in $V$. cholerae. After treatment of bacteria with a sublethal concentration of LL-37 and fractionating into soluble compartments, LL-37 was detected in both the cytoplasmic and the periplasmic space (Figure 6). This suggests that LL-37 is able to enter the periplasm, the likely location in which the interaction between AMPs and SipA occurs. 

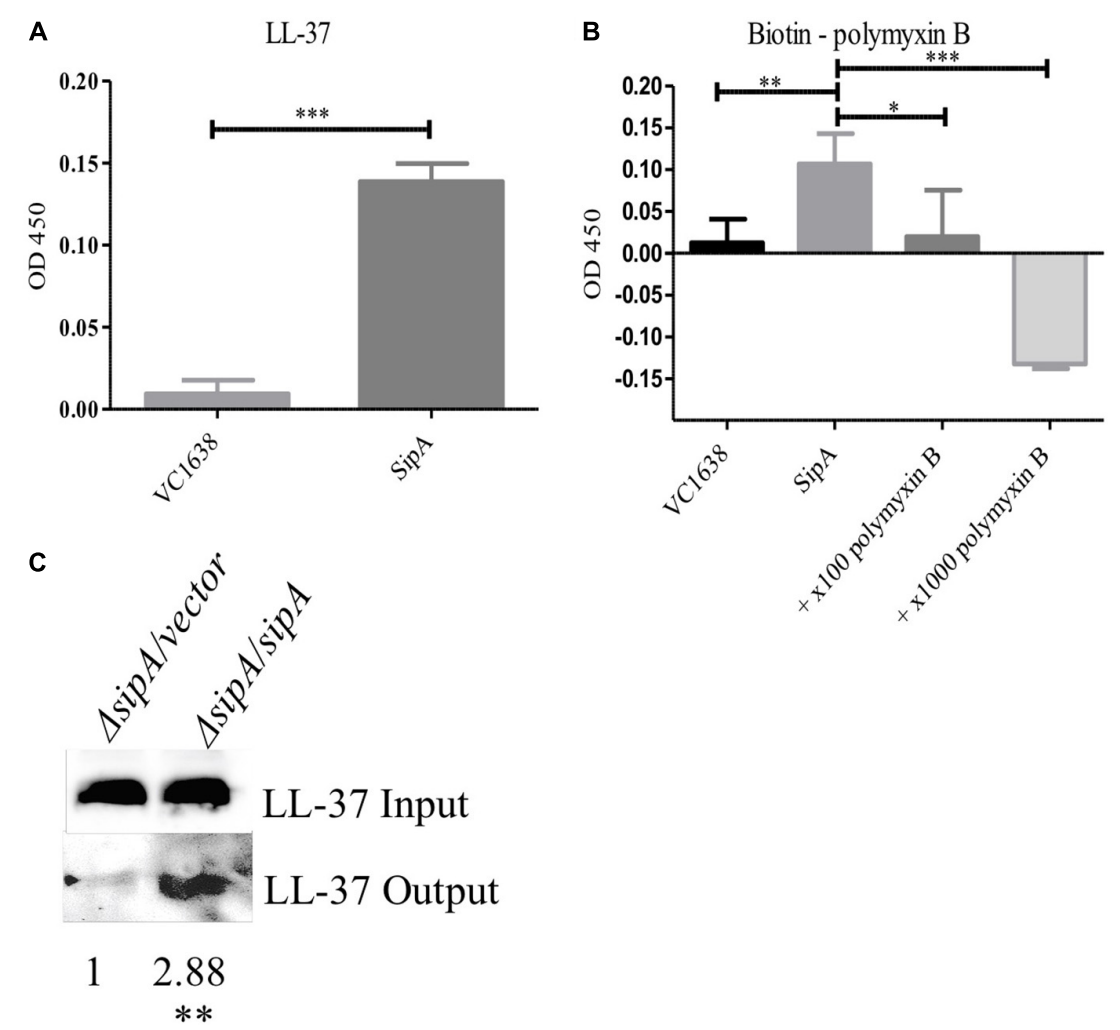

FIGURE 5 | SipA binds polymyxin B and LL-37 in vitro and in vivo. Wells from a 96-well plate were coated with either purified SipA or VC1638 (A,B). LL-37 (A) or biotin-labeled polymyxin B (B) were added to plates and incubated for $1 \mathrm{~h}$. Levels of binding were detected via (A) anti-LL-37 antibody followed by lgG-HRP or (B) Streptavidin-HRP. VC1638 served as a control protein. (C) Coimmunoprecipitation of LL-37 in the presence and absence of $6 \times$ His-sipA. Each culture was crosslinked and lysed before loading onto a Ni-NTA column (input), followed by elution with imidazole (output). Blots were probed with anti-LL-37 antibody. Densitometry of the LL-37 band was calculated based on three separate experiments and normalized to the strain containing empty vector. ${ }^{*} p=<0.05$, ${ }^{* *} p=<0.01,{ }^{* * *} p=<0.001$.

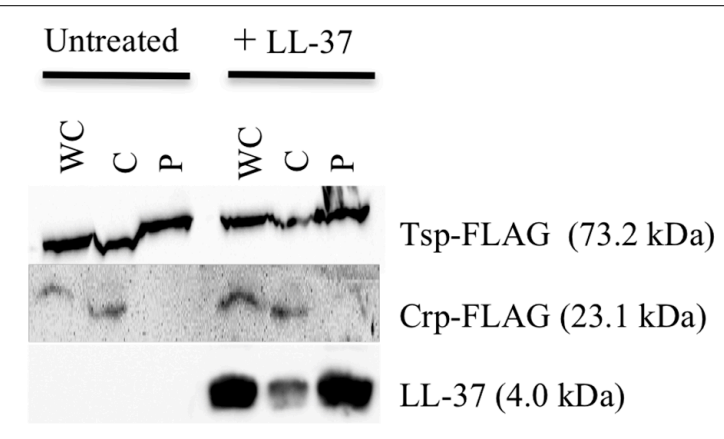

FIGURE 6 | LL-37 is found in the periplasm of $V$. cholerae. Immunoblotting of whole-cell lysates (WC) and soluble fractions from C6707 grown in the presence and absence of LL-37. The membranes were probed with anti-FLAG or anti-LL-37 antibodies. Tsp-FLAG and Crp-FLAG were used as markers for the periplasmic $(P)$ and cytoplasmic $(C)$ fractions, respectively.

\section{SipA Is Required for AMP Resistance in Classical V. cholerae}

One of the more confounding observations from our previous studies on SipA was that a deletion strain did not show increased sensitivity to polymyxin $\mathrm{B}$, in spite of its expression being highly induced by this stress (Matson et al., 2017). However, all of our studies to date have investigated the role of SipA in the El Tor biotype, which is highly resistant to polymyxin B (Roy et al., 1965; Henderson et al., 2017). Classical strains of $V$. cholerae are more sensitive to AMPs due to a frameshift mutation in $\operatorname{alm} F$, a gene in a lipid A modification pathway that is functional in strains of the El Tor biotype (Matson et al., 2010; Samanta et al., 2015). Therefore, we determined if the role of SipA in AMP resistance was more important in this less-resistant background. We first assessed survival of Classical (O395) V. cholerae in the presence of polymyxin B and LL-37 when SipA was overexpressed from a plasmid (Figures 7A,B). The wild type strain (containing empty vector) showed approximately $20 \%$ survival in the presence of $10 \mu \mathrm{g} / \mathrm{mL}$ polymyxin $\mathrm{B}$ and $70 \%$ survival in $5 \mu \mathrm{g} / \mathrm{mL}$ LL-37 after $1 \mathrm{~h}$. Comparatively, a strain overexpressing SipA showed significantly increased survival, approximately twofold in polymyxin B and 1.4-fold in LL-37 (Figures 7A,B). As a control, the periplasmic protein Tsp was also overexpressed and did not increase survival. This suggests that the presence of increased levels of SipA protects $V$. cholerae against AMP killing. To further test the role of SipA in AMP resistance, we generated a sipA mutant in the Classical background of V. cholerae (strain O395). 

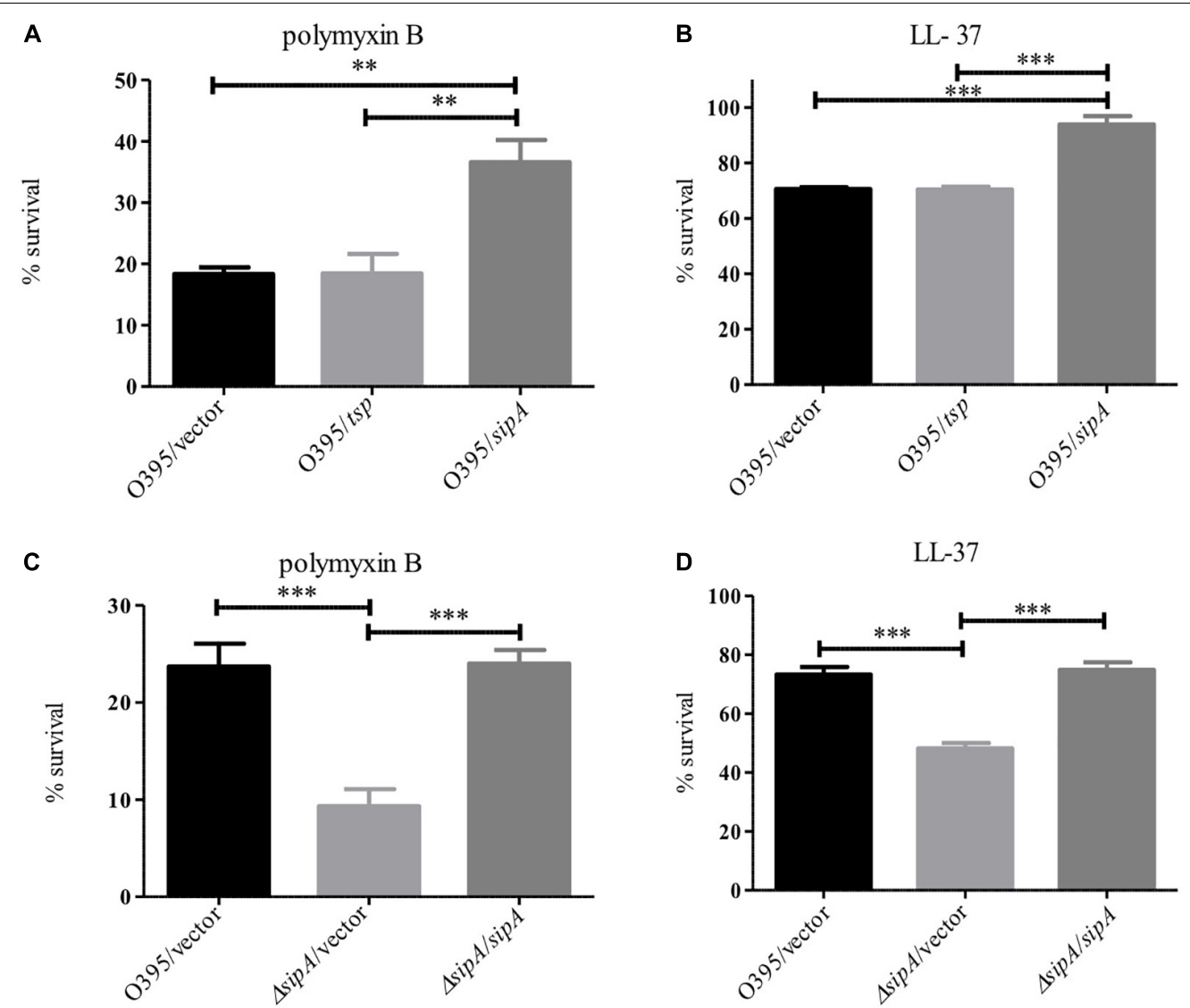

FIGURE 7 | SipA is required for AMP resistance in the Classical biotype of $V$. cholerae. O395 (Classical biotype) overexpressing Tsp or SipA (A,B), and an isogenic $\operatorname{sip} A$ mutant (C,D) were treated with $10 \mu \mathrm{g} / \mathrm{mL}$ polymyxin B (A,C) or $5 \mu \mathrm{g} / \mathrm{mL}$ LL-37 (B,D). After treatment, serial dilutions of each culture were plated for enumeration. Data represents the means and standard deviations of at least three samples. ${ }^{* *} p=<0.01,{ }^{* * *} p=<0.001$.

When the mutant was exposed to polymyxin B or LL-37 it showed a defect in survival that could be restored by complementing with sipA expressed from a plasmid (Figures 7C,D). In order to determine if OmpA also plays a role in AMP resistance in this background, we generated an ompA mutant and tested its ability to survive in the presence of polymyxin B. Similar to a sipA mutant, the strain lacking ompA shows a survival defect in the presence of AMPs (Supplementary Figure S3). These results demonstrate that SipA plays a role in $V$. cholerae surviving AMP stress, and that this role is masked in the highly resistant El Tor background.

\section{DISCUSSION}

Many mechanisms of resistance to AMP stress have been previously described in Gram-negative bacteria, including $V$. cholerae. Despite years of study on this type of stress response, we are still identifying new ways that bacteria respond to insults to their cell envelope. In this study, we describe a new function for a member of the OB-fold family of proteins. While SipA homologs have been shown previously to contribute to AMP, cadmium chloride, and hydrogen peroxide stress
(Pilonieta et al., 2009; Lee et al., 2010), the mechanism of stress relief was unclear. The homologs were shown to interact with various porins, and we similarly found that SipA interacts with OmpA in $V$. cholerae (Figure 3). However, one of the most novel findings of this study was the discovery that SipA interacts with AMPs (Figure 5). This is the first time that a member of the OB-fold family of proteins has been shown to bind AMPs directly, shedding more light on possible functional roles for these proteins.

From our data we predict that SipA/OmpA has a role similar to that of YgiW/OmpF and YdeI/OmpD (Pilonieta et al., 2009); namely that they function together to protect bacteria against cationic AMPs. In addition, we predict that this subfamily of proteins interacts with AMPs in the bacterial periplasmic space. We therefore propose a model where, upon AMP exposure, the VC1638/39 TCS activates expression of sipA. Once made, the SipA protein localizes to the periplasm where it interacts with AMPs that have gained access to the cellular compartment. We also suggest that SipA chaperones the AMPs to OmpA, positioning them for efflux out of the cell (Figure 8). How SipA binds and possibly then releases AMPs is not clear. One could speculate that SipA remains bound to the AMP and exits the cell through OmpA. OmpA is predicted to have a pore 


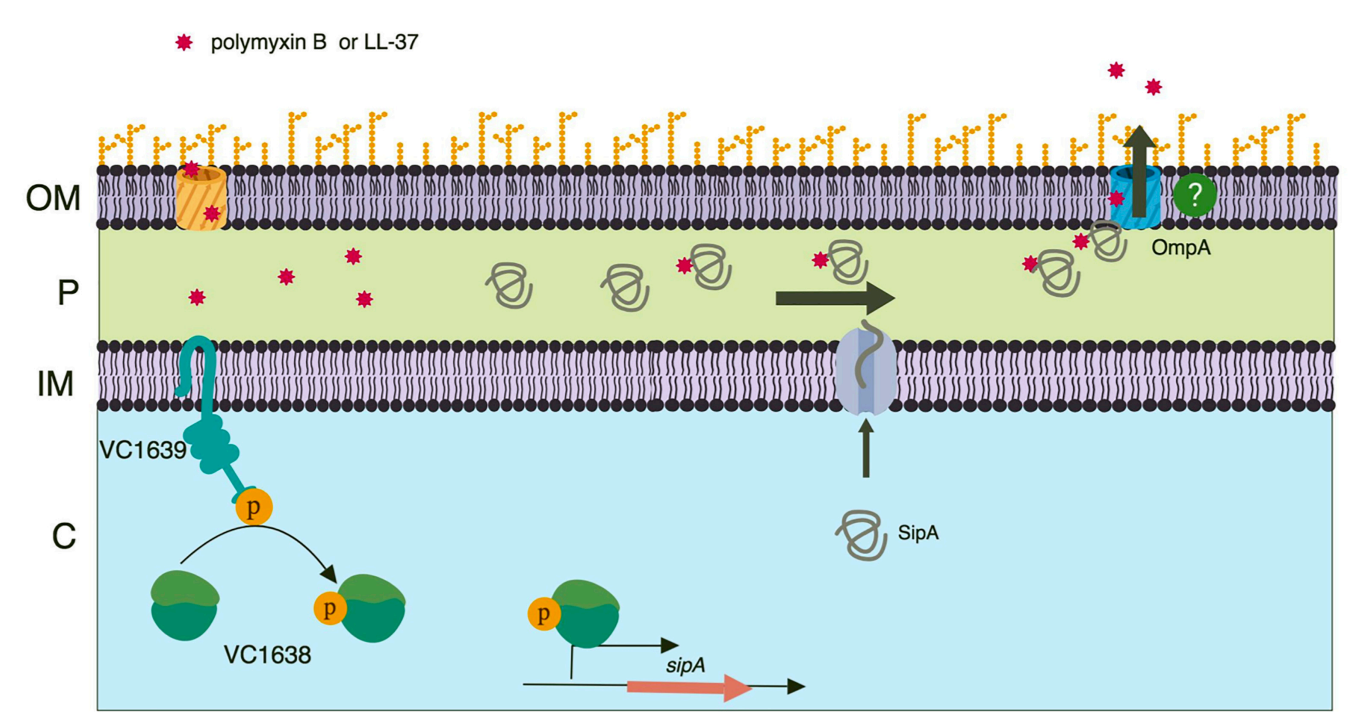

FIGURE 8| Model of SipA-mediated relief from AMP stress. A two-component system, VC1638/39, activates expression of sipA in response AMP exposure. Once made, the SipA protein localizes to the periplasm where it binds AMPs that have entered the bacterial cell. SipA then transports the AMPs to the outer membrane porin OmpA, with which it also interacts. Through an unclear mechanism, the AMPs then exit the cell. While this mechanism is present in both the Classical and El Tor strains of $V$. cholerae, we speculate that AMPs are less likely to enter the bacterial cell in El Tor strains due to the presence of an intact lipid A modification system. Model created using BioRender.

size of 1 to $2.4 \mathrm{~nm}$ in E. coli (Sugawara and Nikaido, 1992; Sun et al., 2013). If $V$. cholerae OmpA has a larger pore size, it is possible that SipA could leave the periplasm in complex with the AMPs. Alternatively, there may be a mechanism by which SipA disassociates with its cargo and remains in the periplasm. This seems more efficient, as unbound SipA would then be able to continue to perform its function if AMPs continue to accumulate in the periplasm. Another possible way that SipA and OmpA could act together to contribute to AMP stress relief is by SipA blocking influx of AMPs into the cell through the OmpA pore. It is possible that SipA interacts with OmpA near the pore, preventing entry of AMPs into the periplasm through the pore by acting as a molecular plug (van der Heijden et al., 2016). Additional studies are needed to determine in more detail how these two proteins act to relieve AMP stress in $V$. cholerae and if these functions are conserved in its homologs.

How SipA and its homologs relieve cadmium chloride and hydrogen peroxide stress is less clear. It is likely that relief from these two stressors uses SipA in a different way than AMPs, as these small molecules seem less likely to directly bind the protein. OmpA appears to play a role in this stress relief pathway as well, as both a single and double mutant (with sipA) show similarly decreased survival in the presence of these stressors. How OmpA works in conjunction with SipA to either rid the periplasm of these compounds, or act to prevent their entry, is unclear.

While cadmium chloride and hydrogen peroxide induce expression of sipA (Supplementary Figure S1), we do not know if that induction requires the VC1638/39 TCS. It is possible that another regulatory network stimulates sipA expression in the presence of other stressors. Of course, we still may identify other stress-inducing agents that require SipA for relief, which may shed more light onto a common mechanistic theme for this pathway.

While we have gained more insight into the function of the SipA protein, there are still additional questions on the upstream regulation of $\operatorname{sip} A$ expression. We have demonstrated that the VC1638/39 TCS is required for sipA expression (Matson et al., 2017), but it is not known if VC1638 directly binds to the sipA promoter, activating its expression, or if there are additional or intermediary transcriptional activators involved. Additionally, we do not know if expression of $v c 1638 / 39$ is conditionally regulated or if it is constitutively expressed. Unlike sipA, our transcriptomic studies showed that polymyxin B exposure did not significantly change expression of either gene of the TCS (Matson et al., 2017). However, it does appear that sipA may be a unique regulatory target of the TCS, as there were no other genes whose expression was induced by polymyxin B and dependent on the presence of the TCS.

One of the most confounding observations going into this study was that a sipA mutant strain did not have a survival defect when exposed to polymyxin B, in spite of its expression being highly induced by AMPs. However, prior to the present study we had only investigated the role of SipA in the highly AMP resistant El Tor biotype of $V$. cholerae (Henderson et al., 2014; Matson et al., 2017). When we tested survival in the Classical biotype, which cannot modify its lipid A, we found that SipA contributes to bacterial survival in the presence of both polymyxin B and LL37 (Figure 7). This suggests that lipid A modification acts as a successful first line of defense against AMPs, and likely prevents a large amount of AMP entry into the cell. In the absence of such 
modification, it is likely that more AMPs enter the cell. In this event, SipA appears to act as an important second line of defense, aiding in the removal of AMPs from the periplasm.

In the Classical biotype survival assays, a sipA mutant shows decreased survival, but a fraction of the population still survives. We therefore hypothesize that in the absence of lipid A modification and sipA, there are yet other mechanisms that protect the bacteria from AMP killing. Gram-negative bacteria have numerous mechanisms to counteract the negative effects of AMPs on cellular functions, and some of these may contribute to survival in the absence of these primary strategies. One mechanism could be through the production of OMVs. OMVs are produced constitutively by Gram-negative bacteria and aid in the elimination of undesired compounds from the cell (McBroom and Kuehn, 2007; Manning and Kuehn, 2011; Schwechheimer and Kuehn, 2013, 2015; Jan, 2017). Additionally, OMVs have a defined role in protection of Gram-negative bacteria against antibiotics and antimicrobials (Kulkarni et al., 2015). Another method of AMP resistance may be their removal through resistance-nodulation-division (RND) efflux systems. These systems work by decreasing the permeability of the outer membrane and regulating efflux of antimicrobials out of the cell. In the absence of RND efflux systems, El Tor strains of $V$. cholerae become 5-fold more sensitive to polymyxin B (Bina et al., 2008) indicating its importance to antimicrobial resistance and suggesting that this may be another layer of protection against AMPs.

Collectively, these observations illustrate the multiple layers of resistance against AMPs in Gram-negative bacteria, and suggest a new resistance mechanism in $V$. cholerae that involves

\section{REFERENCES}

Baba, T., Ara, T., Hasegawa, M., Takai, Y., Okumura, Y., Baba, M., et al. (2006). Construction of Escherichia coli K-12 in-frame, single-gene knockout mutants: the Keio collection. Mol. Syst. Biol. 2:0008. doi: 10.1038/msb4100050

Bahar, A. A., and Ren, D. (2013). Antimicrobial peptides. Pharmaceuticals 6, 1543-1575. doi: 10.3390/ph6121543

Band, V. I., and Weiss, D. S. (2015). Mechanisms of antimicrobial peptide resistance in gram-negative bacteria. Antibiotics 4, 18-41. doi: 10.3390/ antibiotics 4010018

Beck, N. A., Krukonis, E. S., and DiRita, V. J. (2004). TcpH influences virulence gene expression in Vibrio cholerae by inhibiting degradation of the transcription activator TcpP. J. Bacteriol. 186, 8309-8316. doi: 10.1128/JB.186.24.8309-8316. 2004

Bilecen, K., Fong, J. C., Cheng, A., Jones, C. J., Zamorano-Sanchez, D., and Yildiz, F. H. (2015). Polymyxin B resistance and biofilm formation in Vibrio cholerae are controlled by the response regulator CarR. Infect. Immun. 83, 1199-1209. doi: 10.1128/IAI.02700-14

Bina, X. R., Provenzano, D., Nguyen, N., and Bina, J. E. (2008). Vibrio cholerae RND family efflux systems are required for antimicrobial resistance, optimal virulence factor production, and colonization of the infant mouse small intestine. Infect. Immun. 76, 3595-3605. doi: 10.1128/IAI.01620-07

Brownlee, G., and Jones, T. S. (1948). The polymyxins; a related series of antibiotics derived from B. polymyxa. Biochem. J. 43:25.

Carlone, G. M., Thomas, M. L., Rumschlag, H. S., and Sottnek, F. O. (1986). Rapid microprocedure for isolating detergent-insoluble outer membrane proteins from Haemophilus species. J. Clin. Microbiol. 24, 330-332.

Choi, H., Yang, Z., and Weisshaar, J. C. (2017). Oxidative stress induced in E. coli by the human antimicrobial peptide LL-37. PLoS Pathog. 13:e1006481. doi: 10.1371/journal.ppat.1006481 interactions between an OB-fold protein, AMPs, and porins. Inhibition of these systems may have therapeutic benefit, by sensitizing bacteria to host innate immune defenses.

\section{AUTHOR CONTRIBUTIONS}

JS-M and JM conceived and designed the study, wrote the manuscript, and analyzed the data. JS-M performed all the experiments.

\section{FUNDING}

This work was supported by startup funds from the University of Toledo.

\section{ACKNOWLEDGMENTS}

We would like to thank Sarah Plecha and Cara DeAngelis for technical help and critical reading of the manuscript. We would also like to thank Robert Blumenthal for helpful discussions.

\section{SUPPLEMENTARY MATERIAL}

The Supplementary Material for this article can be found online at: https://www.frontiersin.org/articles/10.3389/fmicb. 2019.00161/full\#supplementary-material

Durr, U. H., Sudheendra, U. S., and Ramamoorthy, A. (2006). LL-37, the only human member of the cathelicidin family of antimicrobial peptides. Biochim. Biophys. Acta 1758, 1408-1425. doi: 10.1016/j.bbamem.2006. 03.030

Ginalski, K., Kinch, L., Rychlewski, L., and Grishin, N. V. (2004). BOF: a novel family of bacterial OB-fold proteins. FEBS Lett. 567, 297-301. doi: 10.1016/j. febslet.2004.04.086

Guzman, L. M., Belin, D., Carson, M. J., and Beckwith, J. (1995). Tight regulation, modulation, and high-level expression by vectors containing the arabinose PBAD promoter. J. Bacteriol. 177, 4121-4130. doi: 10.1128/jb.177.14.41214130.1995

Henderson, J. C., Fage, C. D., Cannon, J. R., Brodbelt, J. S., Keatinge-Clay, A. T., and Trent, M. S. (2014). Antimicrobial peptide resistance of Vibrio cholerae results from an LPS modification pathway related to nonribosomal peptide synthetases. ACS Chem. Biol. 9, 2382-2392. doi: 10.1021/cb500438x

Henderson, J. C., Herrera, C. M., and Trent, M. S. (2017). AlmG, responsible for polymyxin resistance in pandemic Vibrio cholerae, is a glycyltransferase distantly related to lipid a late acyltransferases. J. Biol. Chem. 292, 21205-21215. doi: 10.1074/jbc.RA117.000131

Herrera, C. M., Crofts, A. A., Henderson, J. C., Pingali, S. C., Davies, B. W., and Trent, M. S. (2014). The Vibrio cholerae VprA-VprB two-component system controls virulence through endotoxin modification. mBio 5:e2283-14. doi: $10.1128 / \mathrm{mBio} .02283-14$

Herrera, C. M., Hankins, J. V., and Trent, M. S. (2010). Activation of PmrA inhibits LpxT-dependent phosphorylation of lipid a promoting resistance to antimicrobial peptides. Mol. Microbiol. 76, 1444-1460. doi: 10.1111/j.13652958.2010.07150.x

Huntley, J. F., Conley, P. G., Hagman, K. E., and Norgard, M. V. (2007). Characterization of francisella tularensis outer membrane proteins. J. Bacteriol. 189, 561-574. doi: 10.1128/JB.01505-06 
Innis, M. A. (1990). PCR protocols : a guide to methods and applications. San Diego, CA: Academic Press.

Izadpanah, A., and Gallo, R. L. (2005). Antimicrobial peptides. J. Am. Acad. Dermatol. 52(3 Pt 1), 381-390; quiz 391-382. doi: 10.1016/j.jaad.2004. 08.026

Jan, A. T. (2017). Outer Membrane Vesicles (OMVs) of gram-negative bacteria: a perspective update. Front. Microbiol. 8:1053. doi: 10.3389/fmicb.2017. 01053

Joelsson, A., Kan, B., and Zhu, J. (2007). Quorum sensing enhances the stress response in Vibrio cholerae. Appl. Environ. Microbiol. 73, 3742-3746. doi: 10. 1128/AEM.02804-06

Kulkarni, H. M., Nagaraj, R., and Jagannadham, M. V. (2015). Protective role of E. coli outer membrane vesicles against antibiotics. Microbiol. Res. 181, 1-7. doi: 10.1016/j.micres.2015.07.008

Lee, J., Hiibel, S. R., Reardon, K. F., and Wood, T. K. (2010). Identification of stressrelated proteins in Escherichia coli using the pollutant cis-dichloroethylene. J. Appl. Microbiol. 108, 2088-2102. doi: 10.1111/j.1365-2672.2009. 04611.x

Lin, M. F., Tsai, P. W., Chen, J. Y., Lin, Y. Y., and Lan, C. Y. (2015). OmpA binding mediates the effect of antimicrobial peptide LL-37 on Acinetobacter baumannii. PLoS One 10:e0141107. doi: 10.1371/journal.pone.0141107

Linn, T., and St Pierre, R. (1990). Improved vector system for constructing transcriptional fusions that ensures independent translation of lacZ. J. Bacteriol. 172, 1077-1084. doi: 10.1128/jb.172.2.1077-1084.1990

Manning, A. J., and Kuehn, M. J. (2011). Contribution of bacterial outer membrane vesicles to innate bacterial defense. BMC Microbiol. 11:258. doi: 10.1186/14712180-11-258

Matson, J. S., and DiRita, V. J. (2005). Degradation of the membrane-localized virulence activator TcpP by the YaeL protease in Vibrio cholerae. Proc. Natl. Acad. Sci. U.S.A. 102, 16403-16408. doi: 10.1073/pnas.0505818102

Matson, J. S., Livny, J., and DiRita, V. J. (2017). A putative Vibrio cholerae twocomponent system controls a conserved periplasmic protein in response to the antimicrobial peptide polymyxin B. PLoS One 12:e0186199. doi: 10.1371/ journal.pone.0186199

Matson, J. S., Yoo, H. J., Hakansson, K., and Dirita, V. J. (2010). Polymyxin B resistance in El Tor Vibrio cholerae requires lipid acylation catalyzed by MsbB. J. Bacteriol. 192, 2044-2052. doi: 10.1128/JB.00023-10

McBroom, A. J., and Kuehn, M. J. (2007). Release of outer membrane vesicles by gram-negative bacteria is a novel envelope stress response. Mol. Microbiol. 63, 545-558. doi: 10.1111/j.1365-2958.2006.05522.x

Miller, J. H. (1972). Experiments in molecular genetics. Cold Spring Harbor, NY: Cold Spring Harbor Laboratory Press.

Nizet, V. (2006). Antimicrobial peptide resistance mechanisms of human bacterial pathogens. Curr. Issues Mol. Biol. 8, 11-26.

Petiti, M., Houot, L., and Duche, D. (2017). Cell fractionation. Methods Mol. Biol. 1615, 59-64. doi: 10.1007/978-1-4939-7033-9_3

Pilonieta, M. C., Erickson, K. D., Ernst, R. K., and Detweiler, C. S. (2009). A protein important for antimicrobial peptide resistance, YdeI/OmdA, is in the periplasm and interacts with OmpD/NmpC. J. Bacteriol. 191, 7243-7252. doi: 10.1128/JB. 00688-09

Roy, C., Mridha, K., and Mukerjee, S. (1965). Action of Polymyxin of cholera vibrios. techniques of determination of polymyxin-sensitivity. Proc. Soc. Exp. Biol. Med. 119, 893-896. doi: 10.3181/00379727-119-30329

Samanta, P., Ghosh, P., Chowdhury, G., Ramamurthy, T., and Mukhopadhyay, A. K. (2015). Sensitivity to polymyxin B in El Tor Vibrio cholerae O1 strain, Kolkata, India. Emerg. Infect. Dis. 21, 2100-2102. doi: 10.3201/eid2111.15 0762

Schwechheimer, C., and Kuehn, M. J. (2013). Synthetic effect between envelope stress and lack of outer membrane vesicle production in Escherichia coli. J. Bacteriol. 195, 4161-4173. doi: 10.1128/JB.02192-12

Schwechheimer, C., and Kuehn, M. J. (2015). Outer-membrane vesicles from gramnegative bacteria: biogenesis and functions. Nat. Rev. Microbiol. 13, 605-619. doi: $10.1038 /$ nrmicro3525

Skorupski, K., and Taylor, R. K. (1996). Positive selection vectors for allelic exchange. Gene 169, 47-52. doi: 10.1016/0378-1119(95)00793-8

Sochacki, K. A., Barns, K. J., Bucki, R., and Weisshaar, J. C. (2011). Real-time attack on single Escherichia coli cells by the human antimicrobial peptide LL-37. Proc. Natl. Acad. Sci. U.S.A. 108, E77-E81. doi: 10.1073/pnas.1101130108

Sorensen, O. E., Borregaard, N., and Cole, A. M. (2008). Antimicrobial peptides in innate immune responses. Contrib. Microbiol. 15, 61-77. doi: 10.1159/ 000136315

Sugawara, E., and Nikaido, H. (1992). Pore-forming activity of OmpA protein of Escherichia coli. J. Biol. Chem. 267, 2507-2511.

Sun, D., Wang, B., Zhu, L., Chen, M., and Zhan, L. (2013). Block and boost DNA transfer: opposite roles of OmpA in natural and artificial transformation of Escherichia coli. PLoS One 8:e59019. doi: 10.1371/journal.pone.0059019

Teoh, W. P., Matson, J. S., and DiRita, V. J. (2015). Regulated intramembrane proteolysis of the virulence activator TcpP in Vibrio cholerae is initiated by the tail-specific protease (Tsp). Mol. Microbiol. 97, 822-831. doi: 10.1111/mmi. 13069

van der Heijden, J., Reynolds, L. A., Deng, W., Mills, A., Scholz, R., Imami, K., et al. (2016). Salmonella rapidly regulates membrane permeability to survive oxidative stress. mBio 7:e1238-16. doi: 10.1128/mBio.01238-16

Wu, Y., Li, Q., and Chen, X. Z. (2007). Detecting protein-protein interactions by far western blotting. Nat. Protoc. 2, 3278-3284. doi: 10.1038/nprot.2007.459

Conflict of Interest Statement: The authors declare that the research was conducted in the absence of any commercial or financial relationships that could be construed as a potential conflict of interest.

Copyright (C) 2019 Saul-McBeth and Matson. This is an open-access article distributed under the terms of the Creative Commons Attribution License (CC BY). The use, distribution or reproduction in other forums is permitted, provided the original author(s) and the copyright owner(s) are credited and that the original publication in this journal is cited, in accordance with accepted academic practice. No use, distribution or reproduction is permitted which does not comply with these terms. 